\title{
Commutative and Bounded BE-algebras
}

\author{
Zekiye Çiloğlu and Yılmaz Çeven \\ Department of Mathematics, Faculty of Arts and Sciences, Süleyman Demirel University, 32260 Isparta, Turkey
}

Correspondence should be addressed to Yılmaz Çeven; yilmazceven@sdu.edu.tr

Received 2 August 2013; Accepted 28 October 2013

Academic Editor: Yao-Zhong Zhang

Copyright ( 2013 Z. Çiloğlu and Y. Çeven. This is an open access article distributed under the Creative Commons Attribution License, which permits unrestricted use, distribution, and reproduction in any medium, provided the original work is properly cited.

We introduce the notions of the commutative and bounded BE-Algebras. We give some related properties of them.

\section{Introduction}

Imai and Iséki introduced two classes of abstract algebras called BCK-algebras and BCI-algebras $[1,2]$. It is known that the class of BCK-algebras is a proper subclass of BCIalgebras. In [3, 4], $\mathrm{Hu}$ and $\mathrm{Li}$ introduced a wide class of abstract algebras called BCH-algebras. They have shown that the class of $\mathrm{BCI}$-algebras is a proper subclass of $\mathrm{BCH}$ algebras. Neggers and Kim [5] introduced the notion of dalgebras which is another generalization of BCK-algebras, and also they introduced the notion of B-algebras [6, 7]. Jun et al. [8] introduced a new notion called $\mathrm{BH}$-algebra which is another generalization of $\mathrm{BCH} / \mathrm{BCI} / \mathrm{BCK}$-algebras. Walendziak obtained some equivalent axioms for B-algebras [9]. C. B. Kim and H. S. Kim [10] introduced the notion of BM-algebra which is a specialization of B-algebras. They proved that the class of BM-algebras is a proper subclass of $\mathrm{B}$-algebras and also showed that a BM-algebra is equivalent to a 0 -commutative B-algebra. In [11], H. S. Kim and Y. H. Kim introduced the notion of BE-algebra as a generalization of a BCK-algebra. Using the notion of upper sets they gave an equivalent condition of the filter in BE-algebras. In $[12,13]$, Ahn and So introduced the notion of ideals in BE-algebras and proved several characterizations of such ideals. Also they generalized the notion of upper sets in BE-algebras and discussed some properties of the characterizations of generalized upper sets related to the structure of ideals in transitive and self-distributive BE-algebras. In [14], Ahn et al. introduced the notion of terminal section of BE-algebras and provided the characterization of the commutative BEalgebras.
In this paper we introduce the notion of bounded BEalgebras and investigate some properties of them.

\section{Preliminaries}

Definition 1 (see [11]). An algebra $(X ; *, 1)$ of type $(2,0)$ is called a BE-algebra if, for all $x, y$, and $z$ in $X$,

(BE1) $x * x=1$,

(BE2) $x * 1=1$,

(BE3) $1 * x=x$,

(BE4) $x *(y * z)=y *(x * z)$.

In $X$, a binary relation " $\leq$ " is defined by $x \leq y$ if and only if $x * y=1$.

Example 2 (see [11]). Let $X=\{1, a, b, c, d, 0\}$ be a set with the following table:

\begin{tabular}{l|llllll}
$*$ & 1 & $a$ & $b$ & $c$ & $d$ & 0 \\
\hline 1 & 1 & $a$ & $b$ & $c$ & $d$ & 0 \\
$a$ & 1 & 1 & $a$ & $c$ & $c$ & $d$ \\
$b$ & 1 & 1 & 1 & $c$ & $c$ & $c$ \\
$c$ & 1 & $a$ & $b$ & 1 & $a$ & $b$ \\
$d$ & 1 & 1 & $a$ & 1 & 1 & $a$ \\
0 & 1 & 1 & 1 & 1 & 1 & 1
\end{tabular}

Then $(X ; *, 1)$ is a BE-algebra.

Definition 3. A BE-algebra $(X ; *, 1)$ is said to be selfdistributive if $x *(y * z)=(x * y) *(x * z)$ for all $x, y$, and $z \in X$. 
Example 4 (see [11]). Let $X=\{1, a, b, c, d\}$ be a set with the following table:

\begin{tabular}{l|lllll}
$*$ & 1 & $a$ & $b$ & $c$ & $d$ \\
\hline 1 & 1 & $a$ & $b$ & $c$ & $d$ \\
$a$ & 1 & 1 & $b$ & $c$ & $d$ \\
$b$ & 1 & $a$ & 1 & $c$ & $c$ \\
$c$ & 1 & 1 & $b$ & 1 & $b$ \\
$d$ & 1 & 1 & 1 & 1 & 1
\end{tabular}

Then $(X ; *, 1)$ is a self-distributive BE-algebra.

Proposition 5 (see [14]). Let $X$ be a self-distributive $B E$ algebra. If $x \leq y$, then, for all $x, y$, and $z$ in $X$, the following inequalities hold:

(i) $z * x \leq z * y$,

(ii) $y * z \leq x * z$.

Definition 6 (see [15]). A dual BCK-algebra is an algebra $(X ; *, 1)$ of type $(2,0)$ satisfying $(\mathrm{BE} 1)$ and $(\mathrm{BE} 2)$ and the following axioms:

$$
\begin{aligned}
& (\mathrm{dBCK} 1) x * y=y * x=1 \text { implies } x=y, \\
& (\mathrm{dBCK} 2)(x * y) *((y * z) *(x * z))=1, \\
& (\mathrm{dBCK} 3) x *((x * y) * y)=1 .
\end{aligned}
$$

Proposition 7 (see [16]). Any dual BCK-algebra is a BEalgebra. [16].

The converse of Proposition 7 does not hold in general

Definition 8 (see [16]). Let $X$ be a BE-algebra or dual BCKalgebra. $X$ is said to be commutative if the following identity holds:

(C) $x \vee_{B} y=y \vee_{B} x$, where $x \vee_{B} y=(y * x) * x$

for all $x, y \in X$.

Theorem 9 (see [16]). If $X$ is a commutative BE-algebra, then $(X ; *, 1)$ is a dual BCK-algebra.

Corollary 10 ([16]). $X$ is a commutative BE-algebra if and only if it is a commutative dual BCK-algebra.

If $X$ is a commutative BE-algebra, then the relation " $\leq$ " is a partial order on $X[16]$.

In the following, we abbreviate $\vee_{B}$ as $\vee$.

\section{Bounded BE-Algebras}

The following definition introduces the notion of boundedness for BE-algebras.

Definition 11. Let $X$ be a BE-algebra. If there exists an element 0 satisfying $0 \leq x$ (or $0 * x=1)$ for all $x \in X$, then the element " 0 " is called unit of $X$. A BE-algebra with unit is called a bounded BE-algebra.
In a bounded BE-algebra, $x * 0$ denoted by $x N$.

Example 12. The BE-algebra in Example 2 is a bounded BEalgebra and its unit element is 0 .

Example 13. The BE-algebra in Example 4 is a bounded BEalgebra and its unit element is $d$.

Example 14. Let $X=\{1, a, b, c, d\}$ be a set with the following table:

\begin{tabular}{l|lllll}
$*$ & 1 & $a$ & $b$ & $c$ & $d$ \\
\hline 1 & 1 & $a$ & $b$ & $c$ & $d$ \\
$a$ & 1 & 1 & $b$ & $c$ & $d$ \\
$b$ & 1 & 1 & 1 & $c$ & $d$ \\
$c$ & 1 & 1 & 1 & 1 & $d$ \\
$d$ & 1 & 1 & $b$ & $c$ & 1
\end{tabular}

It is clear that $X$ is a BE-algebra, but it is not a bounded BEalgebra.

Theorem 15. In a bounded BE-algebra with unit 0 , the following properties hold for all $x, y \in X$ :

(i) $1 \mathrm{~N}=0,0 \mathrm{~N}=1$,

(ii) $x \leq x N N$,

(iii) $x * y N=y * x N$,

(iv) $0 \vee x=x N N, x \vee 0=x$.

Proof. (i) Using (BE3) and (BE1), we have $1 N=1 * 0=0$ and $0 N=0 * 0=1$.

(ii) Since

$$
\begin{aligned}
x * x N N & =x *((x * 0) * 0) \\
& =(x * 0) *(x * 0) \\
& =1,
\end{aligned}
$$

by (BE4) and (BE1), we get $x \leq x N N$.

(iii) Using (BE4), we have

$$
\begin{aligned}
x * y N & =x *(y * 0) \\
& =y *(x * 0) \\
& =y * x N
\end{aligned}
$$

(iv) By routine operations, we have $0 \vee x=(x * 0) * 0=$ $x N N$ and $x \vee 0=(0 * x) * x=1 * x=x$.

Theorem 16. In a bounded and self-distributive BE-algebra with unit 0 , the following properties hold for all $x, y \in X$ :

(i) $x * y \leq y \mathrm{~N} * x \mathrm{~N}$,

(ii) $x \leq y$ implies $y N \leq x N$. 
Proof. (i) Since

$$
\begin{aligned}
(x * y) & *(y N * x N) \\
& =(x * y) *((y * 0) *(x * 0)) \\
& =(y * 0) *((x * y) *(x * 0)) \quad(\text { by BE} 4) \\
& =(y * 0) *(x *(y * 0)) \quad \text { (by distributivity) } \\
& =x *((y * 0) *(y * 0)) \quad(\text { by BE} 4) \\
& =x * 1 \quad(\text { by BE} 1) \\
& =1 \quad(\text { by BE2 }),
\end{aligned}
$$

we have $x * y \leq y N * x N$

(ii) It is trivial by Proposition 5.

Proposition 17. Let $X$ be a BE-algebra. Then $x * y \leq(y \vee x) *$ $y$.

Proof. Since

$$
\begin{aligned}
(x * y) *((y \vee x) * y) & =(y \vee x) *((x * y) * y) \\
& =(y \vee x) *(y \vee x) \\
& =1,
\end{aligned}
$$

we have $x * y \leq(y \vee x) * y$.

Proposition 18. Let $X$ be a self-distributive BE-algebra. Then, the next properties are valid for all $x, y \in X$ :

(i) $(y \vee x) * y \leq x * y$,

(ii) $x *(x * y)=x * y$.

Proof. (i) Since

$$
\begin{aligned}
x *(y \vee x) & =x *((x * y) * y) \\
& =(x * y) *(x * y) \\
& =1,
\end{aligned}
$$

we have $x \leq y \vee x$. By Proposition 5(i), we have $(y \vee x) * y \leq$ $x * y$.

(ii) Using Definition 3, (BE1) and (BE3), we have

$$
\begin{aligned}
x *(x * y) & =(x * x) *(x * y) \\
& =1 *(x * y) \\
& =x * y .
\end{aligned}
$$

Corollary 19. If $X$ is a self-distributive and commutative BEalgebra, then $(y \vee x) * y=x * y$.

Proof. It is clear by Propositions 17 and 18 and the property (dBCK1).
Corollary 20. If $X$ is a self-distributive, commutative, and bounded BE-algebra with unit 0 , then $x N N N=x N$.

Proof. In Corollary 19, taking $y=0$, we have $(0 \vee x) * 0=x * 0$. Then we get $((x * 0) * 0) * 0=x * 0$; that is, $x N N N=x N$.

Definition 21. In a bounded BE-algebra, the element $x$ such that $x N N=x$ is called an involution.

Let $S(X)=\{x \in X: x N N=x\}$ where $X$ is a bounded BEalgebra. $S(X)$ is the set of all involutions in $X$. Moreover, since $1 N N=(1 * 0) * 0=0 * 0=1$ and $0 N N=(0 * 0) * 0=1 * 0=0$, we have $0,1 \in S(X)$ and so $S(X) \neq \varnothing$.

Example 22. For the BE-algebra in Example 2, it is clear that $S(X)=X$.

Example 23. For the BE-algebra in Example 4, it is clear that $S(X)=\{1, b, c, d\}$.

Proposition 24. Let $X$ be a bounded BE-algebra with unit 0 and let $S(X)$ be the set of all involutions in $X$. Then, for all $x, y \in$ $S(X)$, the following conditions hold:

(i) $x N N N=x N$

(ii) $x \mathrm{~N} * y=y N * x$.

Proof. (i) The proof is obvious by the definition of $S(X)$.

(ii) By Theorem 15(iii), we have

$$
\begin{aligned}
x N * y & =x N * y N N \\
& =y N * x N N \\
& =y N * x .
\end{aligned}
$$

In a bounded BE-algebra $X$, we denote $x \wedge y=(x N \vee$ $y N) N$ where $x \vee y=(y * x) * x$ for all $x, y \in X$.

Theorem 25. In a bounded and commutative BE-algebra $X$ the following identities hold:

(i) $x N N=x$,

(ii) $x \mathrm{~N} \wedge y \mathrm{~N}=(x \vee y) N$,

(iii) $x N \vee y N=(x \wedge y) N$,

(iv) $x N * y N=y * x$.

Proof. (i) Using (BE3), it is obtained that

$$
\begin{aligned}
x N N & =(x * 0) * 0 \\
& =(0 * x) * x, \quad \text { by commutativity } \\
& =1 * x \\
& =x .
\end{aligned}
$$

(ii) By the definition of " $\wedge$ " and (i), we have that $x N \wedge$ $y N=(x N N \vee y N N) N=(x \vee y) N$. 
(iii) By the definition of " $\wedge$ " and (i), we have that $(x \wedge$ $y) N=(x N \vee y N) N N=x N \vee y N$.

(iv) We have

$$
\begin{aligned}
x N * y N & =(x * 0) *(y * 0) \\
& =y *((x * 0) * 0) \\
& =y *(x N N) \\
& =y * x .
\end{aligned}
$$

Corollary 26. If $X$ is a bounded commutative BE-algebra, then $S(X)=X$.

Definition 27. Each of the elements $a$ and $b$ in a bounded BEalgebra is called the complement of the other if $a \vee b=1$ and $a \wedge b=0$.

Theorem 28. Let $(X ; *, 1)$ be a bounded and commutative BEalgebra. If there exists a complement of any element of $X$, then it is unique.

Proof. Let $x \in X$ and $a, b$ be two complements of $x$. Then we know that $x \wedge a=x \wedge b=0$ and $x \vee a=x \vee b=1$. Also since $x \vee a=(x * a) * a=1$ and $a *(x * a)=x *(a * a)=x * 1=1$, we have $x * a \leq a$ and $a \leq x * a$. So we get $x * a=a$. Similarly $x * b=b$. Hence

$$
\begin{aligned}
a * b & =(x * a) *(x * b) \\
& =(a N * x N) *(b n * x N), \quad \text { by Theorem } 25 \text { (iv) } \\
& =b N *((a N * x N) * x N), \quad \text { by BE-4 } \\
& =b N *(x N \vee a N) \\
& =b N *(x \wedge a) N, \quad \text { by Theorem } 25 \text { (iii) } \\
& =(x \wedge a) * b, \quad \text { by Theorem } 25 \text { (iii) } \\
& =0 * b \\
& =1 .
\end{aligned}
$$

With similar operations, we have $b * a=1$. Hence we obtain $a=b$ which gives that the complement of $x$ is unique.

Note that for a bounded and commutative BE-algebra, an element does not need to have any complement.

Example 29. In Example 2, the complement of $b$ is $c$, but $a$ has no complement in $X$.

Theorem 30. Let $(X ; *, 1)$ be a commutative and bounded BEalgebra. Then the following conditions are equivalent, for all $x$, $y \in X$ :

(i) $x \wedge x N=0$,

(ii) $x N \vee x=1$,

(iii) $x N * x=x$, (iv) $x * x N=x N$,

(v) $x *(x * y)=x * y$.

Proof. (i) $\Rightarrow$ (ii) Let $x \wedge x N=0$. Then it follows that

$x N \vee x=(x N \vee x) N N, \quad$ by Theorem $25(\mathrm{i})$

$$
\begin{aligned}
& =(x N N \wedge x N) N, \quad \text { by Theorem 25 (ii) } \\
& =(x \wedge x N) N, \quad \text { by Theorem 25(i) } \\
& =0 N \\
& =1 .
\end{aligned}
$$

(ii) $\Rightarrow$ (iii) Let $x N \vee x=1$. Then, since $(x N * x) * x=$ $x \vee x N=1$ and $x *(x N * x)=x N *(x * x)=x N * 1=1$, we get $x N * x=x$ by $(\mathrm{dBCK} 1)$.

(iii) $\Rightarrow$ (iv) Let $x N * x=x$. Substituting $x N$ for $x$ and using Theorem 25 (i), we obtain the result.

(iv) $\Rightarrow(\mathrm{v})$ Let $x * x N=x N$. Then we get $y N *(x * x N)=$ $y N * x N$. Hence we have $x *(y N * x N)=y N * x N$. Using Theorem 25(iv), we obtain $x *(x * y)=x * y$.

(v) $\Rightarrow$ (ii) Let $x *(x * y)=x * y$. Then we have

$$
\begin{aligned}
x N \vee x & =(x *(x N)) * x N \\
& =(x *(x * 0)) * x N \\
& =(x * 0) *(x * 0) \\
& =1 .
\end{aligned}
$$

(ii) $\Rightarrow$ (i) Let $x N \vee x=1$. Then we obtain

$$
\begin{aligned}
x N \wedge x & =x N \wedge x N N \\
& =(x \vee x N) N, \quad \text { by Theorem } 25 \text { (ii) } \\
& =1 N \\
& =0 .
\end{aligned}
$$

Note that, if $X$ is a self-distributive BE-algebra, then $x *$ $(x * y)=x * y$ by Proposition 18. In this case, Theorem 30(v) is true. If $X$ is also commutative and bounded, then $x N$ is the complement of $x$ by Theorem 30(i) and (ii).

Now we obtain a bounded BE-algebra from a nonbounded BE-algebra as the following theorem.

Theorem 31. Let $(X ; *, 1)$ be a BE-algebra and $0 \notin X$. Define the operation $\circledast$ on $\bar{X}=X \cup\{0\}$ as follows:

$$
x \circledast y= \begin{cases}x * y & \text { if } x, y \in X, \\ 1 & \text { if } x=0, y \in X, \\ 0 & \text { if } x \in X, y=0, \\ 1 & \text { if } x=y=0 .\end{cases}
$$

Then $(\bar{X} ; \circledast, 1)$ is a bounded BE-algebra with unit 0 . 
Proof. It is clear that $\mathrm{BE} 1, \mathrm{BE} 2$, and $\mathrm{BE} 3$ are satisfied. It suffices to verify BE4. Note that

$$
\begin{aligned}
& \text { if } x=0 \text { and } y, z \in X \text {, then } 0 \circledast(y * z)=1 \text { and } \\
& y \circledast(0 \circledast z)=y \circledast 1=1 \text {; } \\
& \text { if } y=0 \text { and } x, z \in X \text {, then } x \circledast(0 \circledast z)=x \circledast 1=1 \text { and } \\
& 0 \circledast(x \circledast z)=1 \text {; } \\
& \text { if } z=0 \text { and } x, y \in X \text {, then } x \circledast(y \circledast 0)=x \circledast 0=0 \\
& \text { and } y \circledast(x \circledast 0)=y \circledast 0=0 .
\end{aligned}
$$

For the remain situations, it is clearly seen that BE4 is satisfied with similar arguments.

We call the BE-algebra $(\bar{X} ; \circledast, 1)$ in the previous theorem the extension of $(X ; 1, *)$. Note that for the BE-algebra $(\bar{X} ; \circledast, 1)$, if for $1 \neq x \in X$, we have $x \notin S(X)$ since $x N N=$ $(x \circledast 0) \circledast 0=0 * 0=1 \neq x$. Hence $S(X)=\{0,1\}$.

Theorem 32. The extension of a self-distributive BE-algebra $(X ; *, 1)$ is also self-distributive.

Proof. Let $0 \notin X$ and denote $\bar{X}=X \cup\{0\}$. Let $\circledast$ be defined as in Theorem 31. We want to see that $x \circledast(y \circledast z)=(x \circledast y) \circledast(x \circledast z)$ for all $x, y$, and $z \in \bar{X}$. For all $x, y \in X$, we know $x \circledast y=x * y$, $0 \circledast y=1, x \circledast 0=0$, and $0 \circledast 0=1$. So we obtain the following, for all $x, y$, and $z \in X$ :

$$
\begin{aligned}
& 0 \circledast(0 \circledast 0)=(0 \circledast 0) \circledast(0 \circledast 0), \\
& 0 \circledast(y \circledast 0)=(0 \circledast y) \circledast(0 \circledast 0), \\
& x \circledast(0 \circledast 0)=(x \circledast 0) \circledast(x \circledast 0), \\
& 0 \circledast(0 \circledast z)=(0 \circledast 0) \circledast(0 \circledast z), \\
& 0 \circledast(y \circledast z)=(0 \circledast y) \circledast(0 \circledast z), \\
& x \circledast(0 \circledast z)=(x \circledast 0) \circledast(x \circledast z), \\
& x \circledast(y \circledast 0)=(x \circledast y) \circledast(x \circledast 0), \\
& x \circledast(y \circledast z)=(x \circledast y) \circledast(x \circledast z) .
\end{aligned}
$$

The above results show that $(\bar{X} ; \circledast, 1)$ is self-distributive.

Theorem 33. If $(X ; *, 1)$ is a commutative, self-distributive, and bounded BE-algebra, then it is a lattice with respect to $\mathrm{V}$ and $\wedge$ where $x \vee y=y *(y * x)$ and $x \wedge y=(x N \vee y N) N$ for any $x, y \in X$.

Proof. From Theorem 3.6 in [14], we know that $(X ; *, 1)$ is a semilattice with respect to $V$. Then we need to show that the set $\{x, y\}$ for all $x, y \in X$ has a greatest lower bound. We know that $x N \leq x N \vee y N$ and $y N \leq x N \vee y N$. By Proposition 5, we have $(x N \vee y N) N \leq x N N$ and $(x N \vee y N) N \leq y N N$. Since $x N N=x$ and $y N N=y$ by Theorem 25(i), we have $x \wedge y \leq x$ and $x \wedge y \leq y$. So $x \wedge y$ is a lower bound of $x$ and $y$. Next we must show that if $z \leq x$ and $z \leq y$ then $z \leq x \wedge y$. Let $z \leq x$ and $z \leq y$. We have $x N \leq z N$ and $y N \leq z N$ by Proposition 5. Since $X$ is an upper semilattice with respect to $\vee$, then we obtain $x N \vee y N \leq z N$ and hence $z N N \leq(x N \vee y N) N$. So we have $z \leq x \wedge y$. Then $x \wedge y$ is the greatest lower bound of $x$ and $y$. So, we can say that $X$ is a lower semilattice. Consequently $(X, \leq)$ is a lattice with respect to $\vee$ and $\wedge$.

\section{References}

[1] Y. Imai and K. Iséki, "On axiom systems of propositional calculi. XIV," Proceedings of the Japan Academy, vol. 42, pp. 19-22, 1966.

[2] K. Iséki, “On BCI-algebras," Kobe Journal of Mathematics, vol. 8, no. 1, pp. 125-130, 1980.

[3] Q. P. Hu and X. Li, "On BCH-algebras," Kobe Journal of Mathematics, vol. 11, no. 2, part 2, pp. 313-320, 1983.

[4] Q. P. Hu and X. Li, "On proper BCH-algebras," Mathematica Japonica, vol. 30, no. 4, pp. 659-661, 1985.

[5] J. Neggers and H. S. Kim, "Ond-algebras," Mathematica Slovaca, vol. 49, no. 1, pp. 19-26, 1999.

[6] J. Neggers and H. S. Kim, "On B-algebras," Matematichki Vesnik, vol. 54, no. 1-2, pp. 21-29, 2002.

[7] J. Neggers and H. S. Kim, "A fundamental theorem of $B$ homomorphism for B-algebras," International Mathematical Journal, vol. 2, no. 3, pp. 215-219, 2002.

[8] Y. B. Jun, E. H. Roh, and H. S. Kim, “On BH-algebras," Scientiae Mathematicae, vol. 1, no. 3, pp. 347-354, 1998.

[9] A. Walendziak, "Some axiomatizations of B-algebras," Mathematica Slovaca, vol. 56, no. 3, pp. 301-306, 2006.

[10] C. B. Kim and H. S. Kim, “On BM-algebras," Scientiae Mathematicae Japonicae, vol. 63, no. 3, pp. 421-427, 2006.

[11] H. S. Kim and Y. H. Kim, "On BE-algebras," Scientiae Mathematicae Japonicae, vol. 66, no. 1, pp. 113-116, 2007.

[12] S. S. Ahn and K. S. So, "On ideals and upper sets in BE-algebras," Scientiae Mathematicae Japonicae, vol. 68, no. 2, pp. 279-285, 2008.

[13] S. S. Ahn and K. S. So, "On generalized upper sets in BEalgebras," Bulletin of the Korean Mathematical Society, vol. 46, no. 2, pp. 281-287, 2009.

[14] S. S. Ahn, Y. H. Kim, and J. M. Ko, "Filters in commutative BEalgebras," Korean Mathematical Society, vol. 27, no. 2, pp. 233242, 2012.

[15] K. H. Kim and Y. H. Yon, "Dual BCK-algebra and MV-algebra," Scientiae Mathematicae Japonicae, vol. 66, no. 2, pp. 247-253, 2007.

[16] A. Walendziak, "On commutative BE-algebras," Scientiae Mathematicae Japonicae, vol. 69, no. 2, pp. 281-284, 2009. 


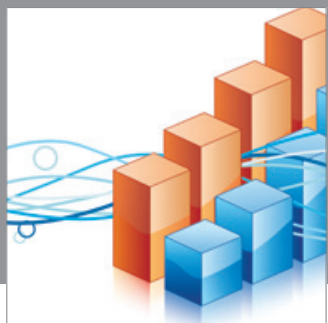

Advances in

Operations Research

mansans

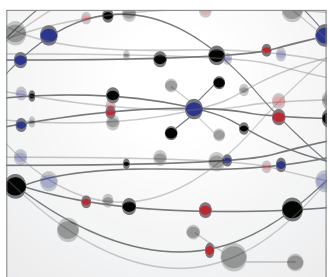

The Scientific World Journal
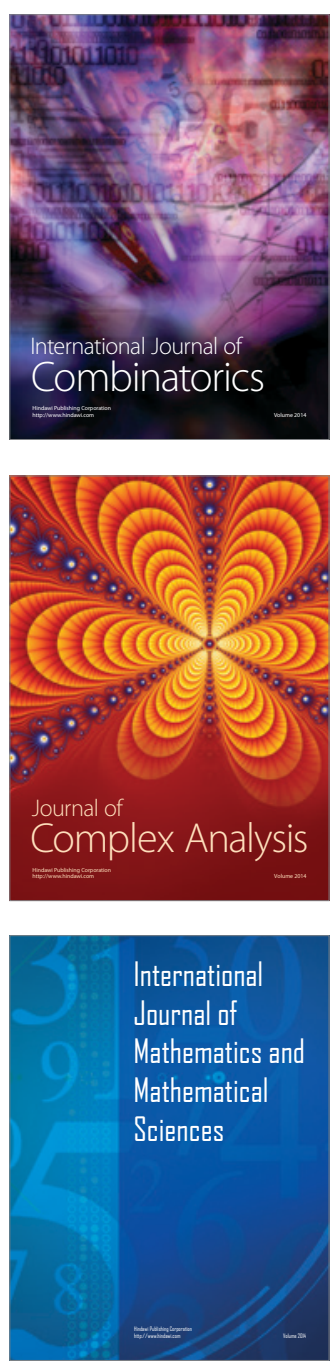
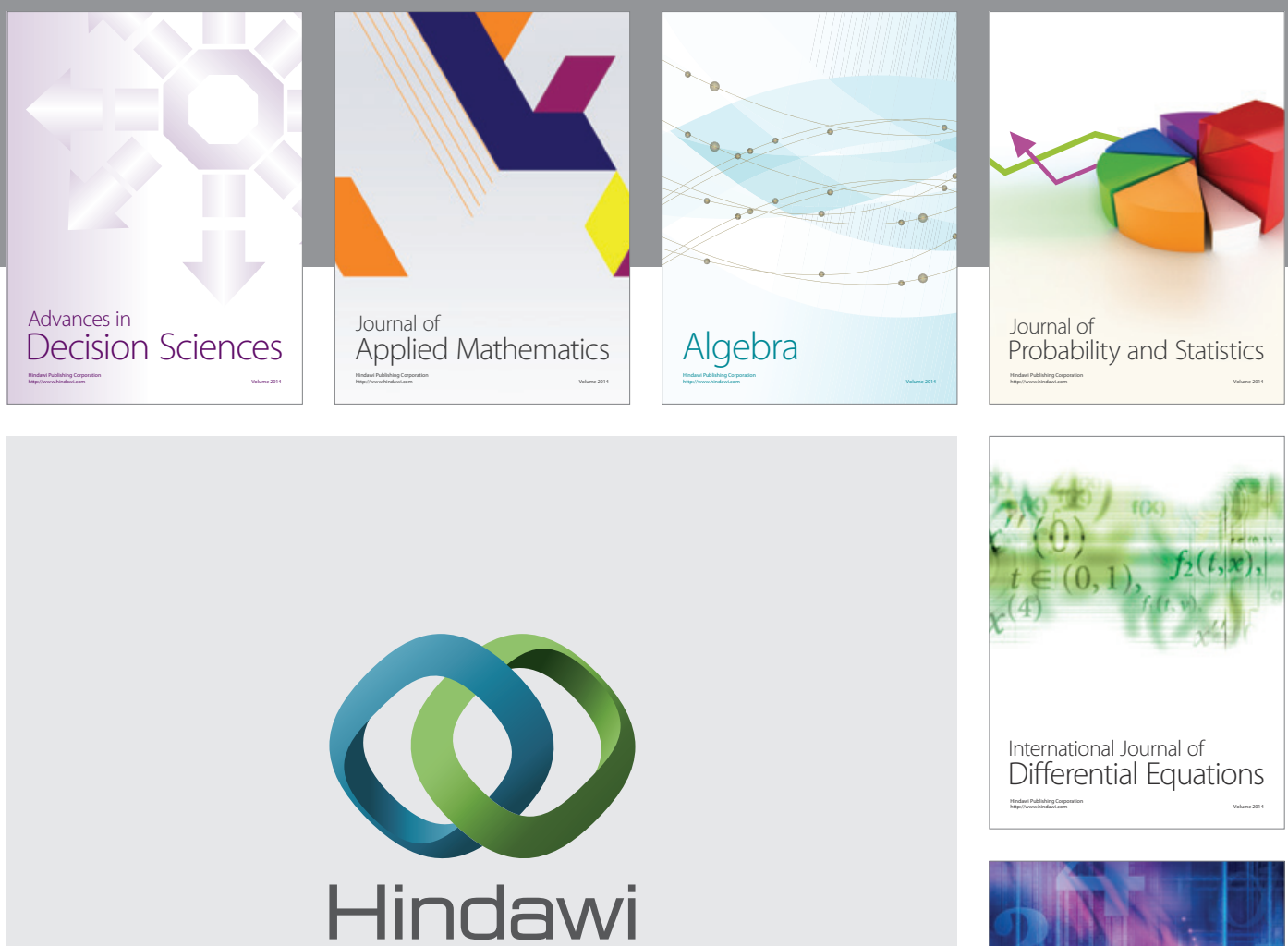

Submit your manuscripts at http://www.hindawi.com
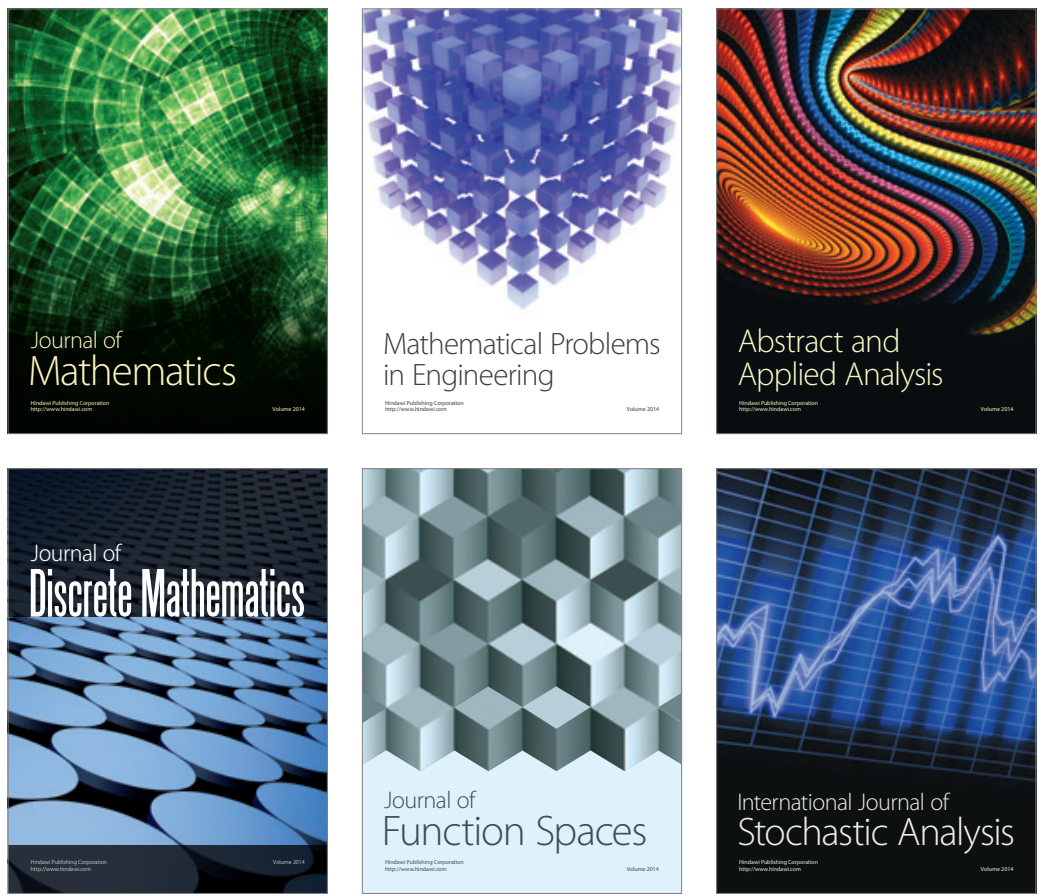

Journal of

Function Spaces

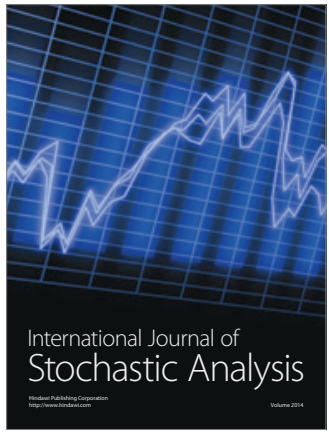

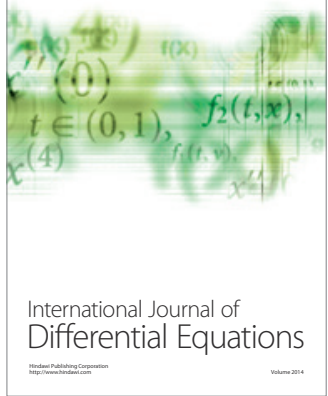
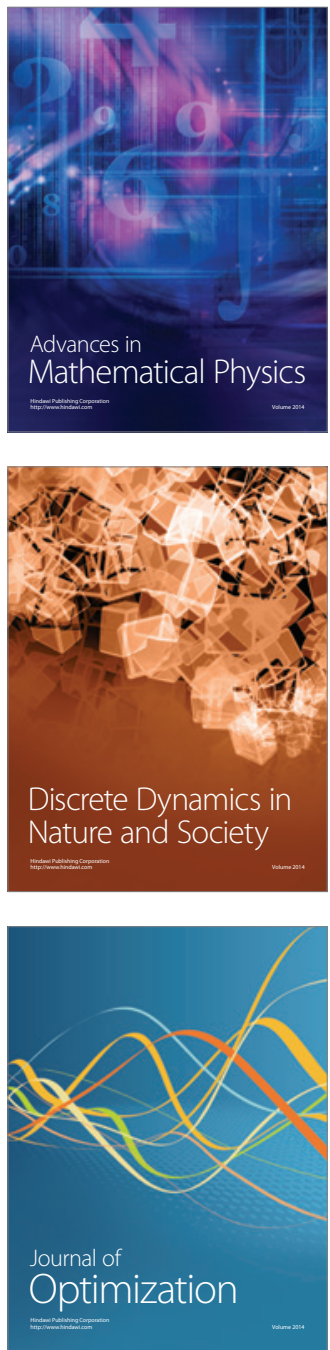